\title{
A Holistic Virtual Laboratory on Wireless Communications and Sensor Networks
}

\author{
http://dx.doi.org/10.3991/ijim.v7i3.2480 \\ Christos K. Liaskos ${ }^{1}$, George C. Koutitas ${ }^{2}$, and Ioannis P. Vlahavas ${ }^{2}$ \\ ${ }^{1}$ Aristotle University of Thessaloniki, Thessaloniki, Greece \\ ${ }^{2}$ International Hellenic University, Thessaloniki, Greece
}

\begin{abstract}
Virtual laboratories have evolved into an adequately mature educational tool for multiple fields of study. Their use is especially beneficial to modern topics such as modern wireless communications. Related solutions in this field explored various approaches and architectures in designing such a virtual environment. The presented software package combines these fragmentary conclusions to a holistic and extensible laboratory architecture. Classic and modern topics, such as propagation, green networking, indoor communications and sensor networks are discussed through interactive 2D/3D environments. The student is also introduced to field measurement procedures and ray tracing principles. Statistical assessment in the context of a postgraduate course in wireless communications demonstrates the educational benefits of the approach.
\end{abstract}

Index Terms - virtual laboratory, holistic approach, wireless communications, sensor networks.

\section{INTRODUCTION}

Engineering has been expanding fast in depth and in number of covered fields over the past decades. The production rate of new knowledge raises educational issues, since engineering course curricula and laboratory equipment cannot keep up. Education on wireless communication and sensor networks constitutes a most representative case of the problem. Networking engineers need to get familiar with classic theories, such as the equations of Maxwell and Shannon's theory of channel capacity and their implications. Moreover, node mobility, energy efficient networking, wireless indoor coverage, sensors and quality of service have evolved to such common, everyday terms that a related educational course is prohibited to ignore. However, constant course curricula and lab updates are not economically viable. Therefore, there is a need for a holistic and extensible virtual laboratory that will offer coverage of all critical wireless engineering issues. This paper presents a software package, incorporated to the postgraduate studies of the International Hellenic University, Greece.

A virtual laboratory can be perceived as a simplified simulator of an existing system. A related, albeit different concept is the remote laboratory. In this case, a piece of software provides controlled access to distant equipment over the Web. The presented software is a purely virtual lab that addresses all vital aspects of modern communications and can be deployed in a standalone fashion.

Virtual and remote laboratories have reached a high degree of maturity over the past years. The study of [1] quantified and compared the learning rate induced by vir- tual labs and lectures on computer networking. The results show that virtual labs can indeed achieve at least the same degree of knowledge absorption as a lecture based approach. However, such a favorable outcome implies that a high degree of realism is attained by the virtual lab [2]. The pedagogical content can also be adaptive to the student needs. For example, [3] proposes a self-evaluation scheme that can be used for adapting a laboratory to the student needs on the fly. An underlying virtual machinebased architecture is also assumed. Indicative applications of virtual labs include electronics [4], wired networking [5], renewable sources of energy and e-health [6].

Various, outstanding virtual laboratories on wireless communications and sensor networks have been implemented independently by academic and commercial entities. As expected, however, the achieved topic coverage is fragmentary and overlapping. Thus, covering the contents of a given course would typically require the concurrent adoption of more than one of these laboratories. However, the applications follow different functionality and GUI principles. Thus, familiarizing with several of them, typically in a single semester, would result into an impractically steep learning curve for the students. Furthermore, overlapping topics and lack of interoperability would force a student to pointlessly learn to do the same thing (e.g. setup an antenna) in several GUI environments. Nonetheless, the point of a virtual lab is not to familiarize the students with new software, but rather to help them understand the studied topic in greater depth. The presented software package addresses this issue, providing a holistic coverage of wireless engineering topics in a welldefined, non-overlapping and extensible manner.

The remainder of this paper is organized as follows. Section II studies notable, related virtual laboratories. Section III presents the course curricula targeted by the virtual laboratories. Implementation issues are discussed in Section IV. Student feedback assessment and deployment experiences are described in Section V. Conclusive remarks are given in Section VI. The present work complements the demonstration of by presenting the yearlong statistical assessment of the package, as well as by incorporating the sensor network topic.

\section{RELATED SOFTWARE PACKAGES}

Virtualizing wireless communications systems has gained significant focus from academic and commercial entities. Various software packages have been presented, each with a specific focus. 
Explanation of theoretic modulation and encoding is the strict goal of [7] and [8]. These labs allow the student to form circuits and set driving signals and oscillators, in an effort to visualize the transformation that a piece of information goes through before its wireless propagation. In a similar manner, [9] studies channel modeling alongside modulation and encoding issues, introducing error correction codes as well. The package adopts a textbook based, block diagram interface, commonly found in tools such as MATLAB/SIMULINK. The WiFi Virtual Laboratory of addresses node positioning and networking issues in the context of the IEEE 802.11 standard. The basics of Medium Access Control (MAC) and Transfer Control Protocol (TCP) are examined. In particular, the laboratory visualizes the exchange of Ready-to-Send/Clear-to-Send MAC signals, as well as the TCP handshaking process. A newer iteration of the approach allows for cooperative involvement of multiple users at once. Using commercial simulators -for educational purposes is also a popular approach. These software packages can be used to model any communications system, without restriction. However, they require familiarization with a programming environment designed for specialists. In other words, in depth knowledge of the studied topic is required prior to their use, contradicting to their use as educational tools. Finally, another commercial educational solution, offers technical training by virtualizing actual hardware. Network routers, switches, hubs and cables are fully presented in a $3 \mathrm{D}$ environment. However, no connection to theoretical aspects is provided. This continued fragmentation of topics is in opposition to, which states that there is a need for merging several simulation tools and virtual lab software for a single university course. Most importantly, all related studies, commercial or not, focus on classic issues of wireless communications, disregarding the modern aspects of green engineering, sensor networking and energy efficiency.

Table I compares the features offered by the presented and existing virtual laboratories. The presented package promises much wider topic coverage. This conclusion holds not only for the number of topics, but for the number of studied standards as well. Cellular telephony, wireless LAN and digital television standards are discussed through the various GUIs. It is notable that certain approaches offer greater depth of study in certain topics. For example, [7] and [9] excel at specializing in modulation and encoding issues, as discussed. However, there is no straightforward way for combining the related approaches in one concrete whole. A student or tutor would have to install all packages of interest, get familiar with the different GUIs and extract the useful features.

The presented laboratory is intended as an approach that generalizes and combines the preceding efforts in a well-defined whole. Furthermore, classic and modern issues of wireless communications are covered. Antenna design and positioning channel modeling, adaptive/static modulation, mobility, coverage estimation, networks planning of indoor and terrestrial networks, green communications and sensor networking are thoroughly discussed through intuitive 2D/3D parametrical interfaces. Hierarchical content and persistence of GUI traits throughout the lab makes for a smooth learning curve. The applications take advantage of integrated ray tracing algorithms that have been validated with real measurements to increase the accuracy of the findings. The software pack- age is extensible, free and open source, implemented in plain MATLAB ${ }^{\mathrm{TM}}$ code. The use of alternative platforms (e.g. OCTAVE) is possible, but not actively supported by the authors due to market exposure, extensibility and performance limitations.

TABLE I.

COMPARISON OF VARIOUS VIRTUAL LABORATORIES ON WIRELESS COMMUNICATIONS IN TERMS OF COVERED TOPICS. PLUS SYMBOLS DENOTE THE RELATIVE RANKING IN TERMS OF FEATURES.

\begin{tabular}{|c|c|c|c|c|c|}
\hline Topics covered & IHU Vlabs & [7] & [9] & & [8] \\
\hline Propagation mechanisms & ++ & & & & \\
\hline Antennas & ++ & & & & \\
\hline Node mobility & ++ & & & + & \\
\hline Channel models & ++ & & & & \\
\hline Modulation & + & ++ & ++ & & \\
\hline Encoding & + & & ++ & & + \\
\hline Standards & $\begin{array}{c}\text { GSM, UMTS, } \\
\text { WiFi, DVB }\end{array}$ & & & WiFi & \\
\hline Network Planning & ++ & & & + & \\
\hline Green Networking & ++ & & & & \\
\hline Sensor Networking & ++ & & & & \\
\hline
\end{tabular}

\section{Pedagogical Scope: TARgeted Courses And METHOD OF INCORPORATION}

MSc courses typically incorporate a large diversity of taught modules and students with heterogeneous backgrounds. In addition, their duration is limited, constituting the virtual laboratories significant in the absorption of knowledge. GUI demonstrations and virtual experiments are important for educational purposes since they provide a holistic and an applied view of the taught concepts. This Section presents a real MSc course which incorporated the discussed virtual laboratory in its structure. The MSc modules are introduced, mentioning the related virtual laboratory GUIs. The GUIs themselves are detailed in Section IV.

\section{A. Related MSc Modules}

The following three modules of the MSc in Information and Communication Technology (ICT) Systems of the International Hellenic University are offered as a 19hour core and elective package over a 13 week term. The virtual laboratory acts as a supplement to traditional, classroom teaching, which takes place in parallel over the same period. A complete description of the MSc program curriculum can be found in. The number of enrolled students specializing in communications is typically $15-30$ per year. The virtual laboratories focus on the most critical and difficult to understand subjects. Mobile Communication Networks (9hour): This is a core module in the curricula and explores the functionality layers of wireless networks for different wireless communication platforms such as WiFi, Digital Video Broadcasting (DVB), Long Term Evolution (LTE), WiMax, Bluetooth, adhoc and mobile satellite systems. In addition, network planning aspects of wireless systems are investigated, giving a more industrial perspective to the course. The module concentrates on physical layer aspects, such as channel modeling, modulation coding, antennas and propagation since they typically comprise challenging concepts for the majority of the enrolled students. The virtual labs on wireless 
communications help the student understand the wireless channel under theoretical and virtual drive test procedures (see core vlabs 1, 2, 3, Section IV) and perform her own network planning and testing investigations in terrestrial and indoor networks (see application labs $6-8$ ) Sensor Networks (5hour): This is an elective module in the curricula and explores data communication aspects in sensor networks. The module covers ad hoc, mesh and star topologies and concentrates on energy efficiency and networking issues. Specifically, sensor network topology maintenance and routing algorithms are thoroughly investigated within the context of the module, while the network longevity is a major performance metric. The virtual labs on sensor networks explore both aspects and more precisely vlab 5 investigates network topology control whereas vlab 6 explores routing algorithms. Green ICT (5hour): This is an innovative module offered as an elective module in the curricula. The Green ICT is divided into two main areas. The fist concerns the use of ICT for sustainable growth and better quality of life, focusing on environmental monitoring and participatory sensing. The second part focuses on energy efficiency issues in wired and wireless networks. The environmental impact of green computing and network planning techniques is discussed, focusing on the resulting total $\mathrm{CO} 2$ emissions and grow sustainability. More precisely in vlabs $6-7$ the user has the ability to explore different DVB network planning architectures and observe the total network power consumption and CO2emissions based on empirical models that correlate the base station power consumption with the wireless transmission output. This is important since the base stations in a wireless network are the most energy 'hungry' elements. Furthermore, in vlab 5 the user has the ability to observe energy efficiency and battery level indications in a sensor network under different topologies and this is then used to quantify the network lifetime.

\section{B. Teaching Method}

MSc in ICT Systems favors adaptive self-learning and lifelong education schemes. From the teaching perspective, the virtual laboratories are at first demonstrated in a supervised manner to the students during the semester, at the end of critical module chapters. There are 3 virtual laboratory tutorials per module where the students gain hands on experience within the PC lab of the International Hellenic University (IHU).

It is highlighted that the software package is accompanied by a set of exemplary solved examples as well as unsolved problems. The demonstrator briefly describes the available set of exercises that can be found at the related web site. Then, the students are given a predefined time window (typically a week) to run their own experiments and discuss the conclusions with the tutor. The virtual lab exercises can be a part of the final course mark, depending on the institution and the professors' preferences. Their scope is to stimulate discussion on applied aspects in telecommunications and encourage further studies on the covered topics.

\section{DESIGN AND IMPLEMENTATION}

In accordance with the course curriculum and the general structure of the related textbooks, the laboratory was divided into seven topics. Each topic is treated through a dedicated corresponding application. However, the topics covered are not uncorrelated. Certain basic topics of wire- less communications needed to be covered first. The remaining topics can be perceived as application scenarios that rely on this basic knowledge. Thus, a hierarchical architecture was chosen for the software package. The following three core laboratories deal with basic issues, each encapsulated into the other in the way depicted in Fig. 1. The full, explicit depth and functionality of each lab can be derived from their online documentation. A compact overview follows:

Core lab 1. The lab introduces the students to antenna characteristics, propagation mechanisms and path loss models. The user is able to setup and customize a receiver and a transmitter, regulating transmission power, antenna patterns, orientation and resistance, receiver sensitivity and path loss attributes. The educational goal is the formation of an empirical connection between the setup choices, the resulting power measurements and the achieved wireless coverage. The lab uses discrete event simulation.

Core lab 2. Having studied static topologies, the complete functionality of the first lab is transferred to a mobile setting. In a full 3D environment, the user is able to configure the movement of a vehicle through a simple urban environment. A parametric 3D world introduces the notion of non/line of sight communications, shadowing, fading and noise effects. The 3D world was designed in VRML (Virtual Reality Modeling Language) and is animated through the MATLAB Animation Toolbox. In conjunction with the first core lab, a complete coverage of physical layer issues is achieved (Fig. 2). Once again, the simulation is nonrestrictive, discrete event based.

Core lab 3. The third core lab proceeds to study the effects of encoding and modulation in the context of the GPRS (General Packet Radio Service) and UMTS (Universal Mobile Telecommunications System) standards. Achieved bitrates and call dropping probabilities are studied thoroughly in a mountainous terrain traversed by a vehicle. The lab also introduces the student to field measurement techniques, detailing a realistic equipment setup mounted on the traveling vehicle. Utilizing this fact, the

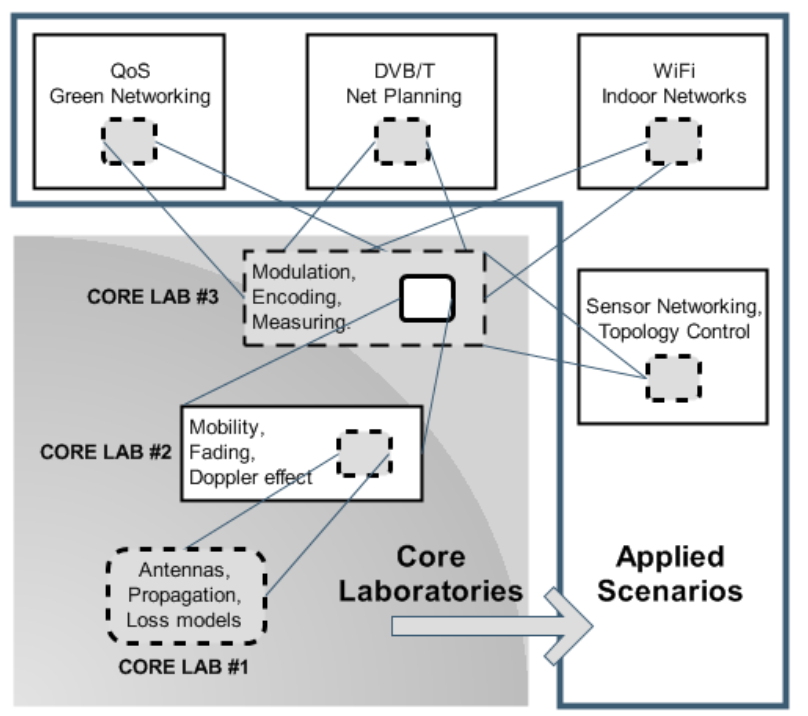

Figure 1. The content of the GUIs comprising the virtual application influences the software architecture as well. The functionality of each core module is incorporated to subsequent applications. The formed parent-child hierarchy means that the laboratory exercises become more advanced, while using already learned GUI elements. 
PAPER

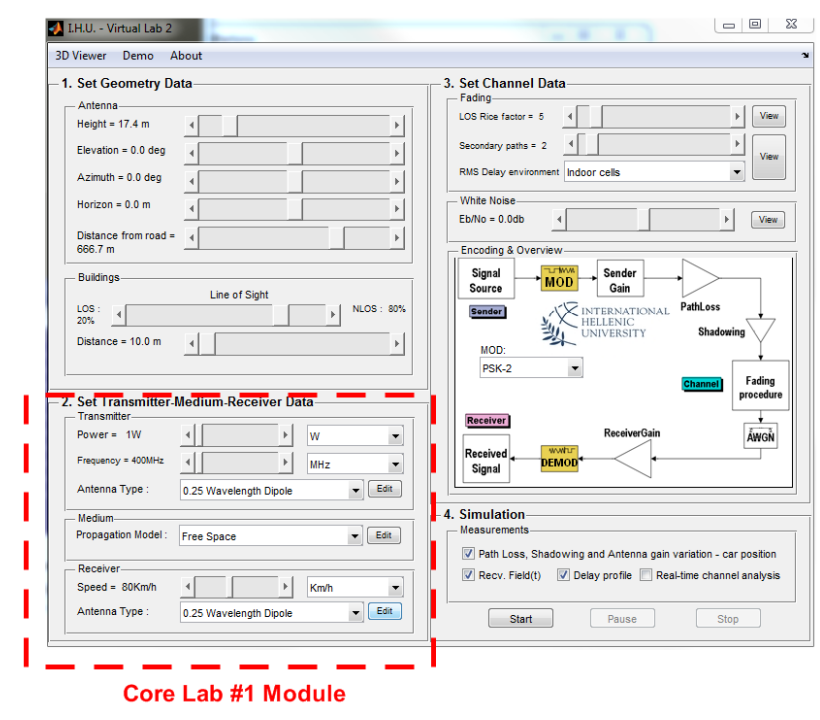

(a) Application of the concept of Fig. 1 to the interface of the second core lab. The annotated panel incorporates the functionality of the first core lab pertaining to antenna patterns, loss models and propagation. The functionality is then extended to cover mobility, fading, noise and simple encoding aspects. A text book styled block diagram of the channel provides connection to theory.

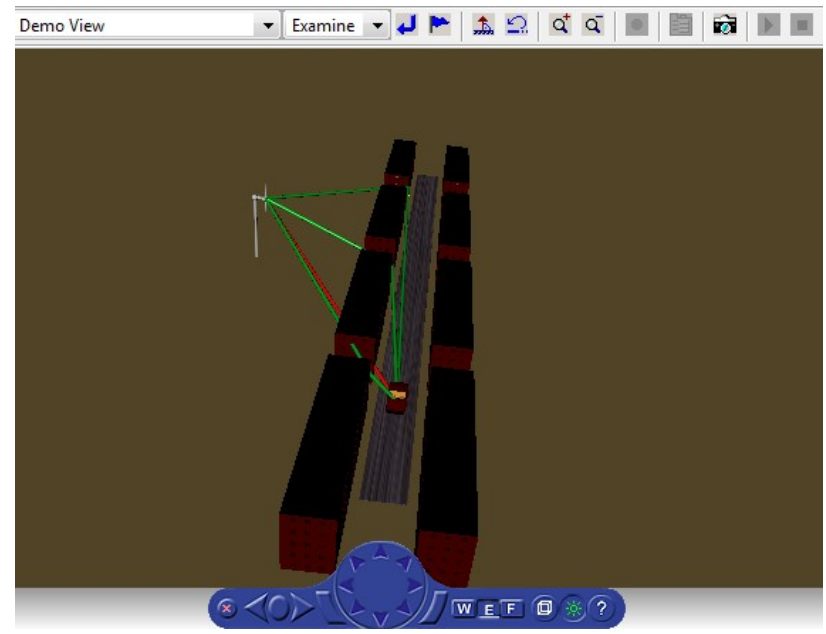

(b) Apart from theoretic representation, the labs offers animated visualizations of the studied phenomena. Thus, a degree of realism is achieved. In the depicted case, the rays propagate on their surroundings, traveling from a transmitter to a vehicle moving along a road.

Figure 2. The GUI of the second core lab as an example of practical application of the design concepts.

student is also introduced to channel measurement setups and statistical channel modeling concepts. This laboratory employs deterministic animations and the student is able to execute any part of the recorded vehicle route. However, the signal reception is simulated (discrete event based) and the student can observe the effects of his choices in the reception quality.

The remaining laboratories are characterized as strongly applied scenarios. They rely on and encapsulate the complete functionality of the three core laboratories and study the following settings. They also serve as examples for building advanced extensions covering modern issues of wireless communications. Full discrete event simulation is used in each lab:

Application lab 4. The laboratory aims to familiarize the student with the issue of balancing QoS and energy efficiency in a network. The setting comprises a real 3D terrain (Geographic Information GIS data of a region of Western Macedonia, Greece). Relying on the experience gained from the core labs, the user is asked to place and configure broadcast transmitters on the terrain in a way that yields acceptable coverage and $\mathrm{CO} 2$ emissions. A software module visualizes signal quality in the form of a still TV image, connecting theory to practice. The user can also explore the effects of different transmission parameters(guard interval, modulation type, etc.) on the provided QoS.

Application lab 5. This laboratory seeks to incorporate research elements in the presented software package. A DVB scenario is assumed over the terrain of the applied lab 1. The transmitter positions may only be placed on mountain tops, as in reality. Furthermore, we assume interest in providing wireless coverage only to populated areas (towns and villages). The advanced student is expected to devise a way of choosing the proper amount and positions of transmitters, while keeping $\mathrm{CO} 2$ emissions in check. In for example, genetic algorithms were successfully adopted for the task.

Application lab 6. Indoor network planning is studied next. The student is familiarized with ray tracing principles and deterministic channel estimation in a WiFi setting. The effects of adaptive modulation are studied in detail. Additionally, the student ma also perform research in minimizing the number of access points required to cover the inner parts of a building. Real floor plans and ray tracing data are supplied. Finally, the user can study narrowband and wideband channel characteristics obtained by the ray tracing code (Rice factor, Delay spread, etc.).

Application Lab 7. Network formation, routing, topology control and maintenance algorithms for sensor networks are studied in the final laboratory. The student can observe the efficiency of well-known topology maintenance algorithms. Algorithm dependent energy consumption is also calculated. The laboratory also serves as a proof of concept for incorporating third party simulators to the presented software package. The routing module stems from, while topology maintenance functions are derived from. A snapshot of the routing module is given in Fig. 3. The topology maintenance module follows the same GUI principles.

\section{A. Educational Principles}

The presented software package aims at providing a holistic coverage of wireless communication topics. The hierarchy of the GUIs promotes the progressive familiarization of the student with theoretic and practical aspects of wireless networking. The study is both qualitative and quantitative. Upon completing a set of exercises, the user is expected to have formed a mental link between a network configuration and the resulting link budget. Furthermore, a connection between a link budget and the ensuing quality of service (QoS) is attempted. For example, the DVB applied lab visualizes the expected video quality for a given Signal-to-Noise level at the receiver. The aforementioned educational goals are thus defined as follows. The user is expected to: a) Perform a holistic study of wireless networking. b) Connect network conditions to rough link budget expectations (quantitative study). c) Correspond link budgets to practical QoS levels (qualitative study). 
Each GUI offers a very high number of possible configurations. The idea is to simulate reality from the aspect of a high number of freedom degrees. The GUIs purposefully provide a system setup that cannot be configured for successful operation in an obvious way. Indeed, a student would require days of work to solve an exercise, should he resolve to "brute forcing" the form controls without understanding their physical meaning and impact. The user is thus forced to gain a theoretical and practical understanding of the inner workings of the simulated system. This approach also serves as a mild introduction to difficulties of setting up a real system.

The quasi-realistic degree of freedom calls for a well

planned series of exercises that guide the user through the simulated environments. These exercises are considered essential to the success of the software package. Starting of with elements of theory, these exercises explore system aspects in an incremental fashion. They also aim at demonstrating the full potential of the GUIs, thus acting as a form of hands on manual as well. The incremental complexity of the exercises is also reflected on the complexity of the corresponding GUI controls.

\section{B. Technical Principles}

As stated, an educational goal of the software package is to connect theory to practice. Block diagrams constitute a classic way of representing wireless channel models and networking issues. Therefore, the GUIs incorporate both block diagram representations and realistic 3D visualizations of the examined scenarios. A typical example of the concept is given in Fig. 2. The user may select the operational attributes and observe the impact on the virtual environment, as well as on the block diagram. Combined with the ensuing measurements, the formation of a mental link between the theoretical representations and the end result is attempted. The packages employ discrete event simulation for their operation, which is derived from MATLAB.

The software package was designed to provide a smooth learning curve. This principle is also evident from the GUI design. Each lab is designed to occupy a single configuration form, providing access to all features in a non-cluttered manner. Simplicity is maintained in the GUI design choices, adopting only classic form controls. Panels group form components by functional purpose. The GUIs are designed to fit nicely in a PC or laptop screen. All aforementioned actions aim at facilitating the submergence of the user into the studied topics, rather than into familiarization with GUI quirks.

A critical technical consideration was extensibility. Apart from the modular design presented in Fig. 1, the software architecture enables the easy addition of new antenna types, measurement configurations and channel models. Exploiting the support of MATLAB for object oriented programming, any such an addition is as easy as extending a predefined class interface. MATLAB code is stored in simple text files. The addition of such files into predefined folders is automatically detected and the GUIs are updated correspondingly. These simple modules, along with cached data files (e.g. ray traced maps and field measurements) are fed to a MATLAB/SIMULINK model that handles the synchronization between user actions and virtual world updates. The virtual worlds themselves are stored in the well-known VRML format (Virtual Reality Modeling Language), enabling straightforward customi-

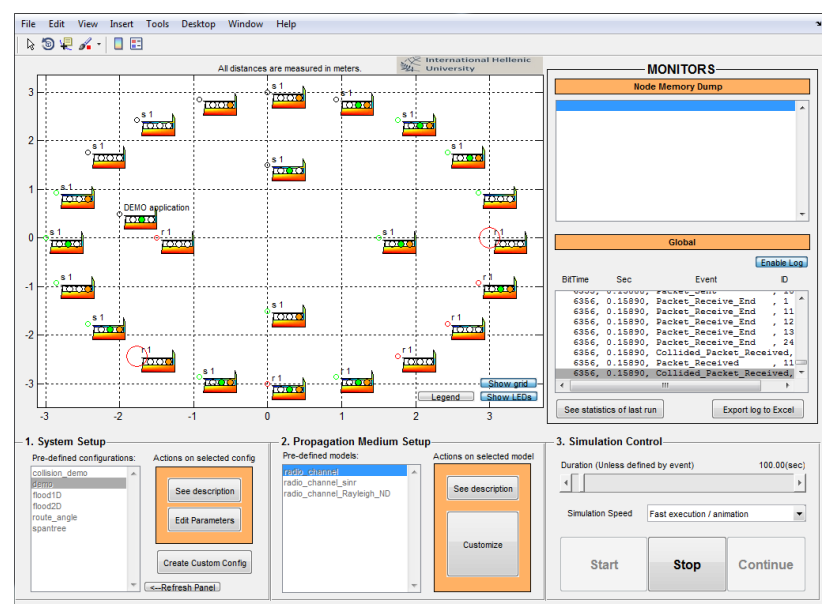

Figure 3. Snapshot of the wireless sensor routing module. The student can set up a topology, either manually or via automatic deployment. Once the physical parameters have been set (e.g. channel type), packet routing events can be observed, logged and statistically studied. Original functionality is derived from.

zation. A laboratory can be fully or partially encapsulated into another by incorporating the corresponding SIMULINK model and associated files, implementing the educational hierarchy of Fig 1.

Finally, proper documentation was considered essential to the software package. The virtual laboratories come with extensive manuals covering the aspects of the developer (architecture and extensibility), the tutor and the student.

\section{Student Feedback Assessment}

The virtual labs were used by postgraduate students of the MSc program in ICT Systems at the International Hellenic University for the academic year $(2011-2012)$. The involved students were asked to provide feedback regarding their overall experience. The goal of the assessment was to hint whether the virtual laboratory succeeded in spurring the students' interest during their studies.

The department tutors were actively involved in the software design and development. Therefore, an objective assessment could only rely on the student experience and the end result (course grades). It is clarified that no claim is made towards quantifying the impact of the laboratories on the course grades. Teaching is a complex process, involving highly variable parameters such as tutor/student commitment and talent. The presented process simply presents data that hint increased student interest due to the use of the laboratory.

At first, volunteers were called to participate in the evaluation process. More than a full month after the end of the course, a number 12 out of the total 20 students exhibited interest in the process. In other words, more than 50\% of the students volunteered without any motive regarding their grades or other. The students filled in a questionnaire regarding their overall experience with the software package. The questionnaire included questions regarding the usefulness of the labs, their ease of use, the GUI environment and the ability to enhance the knowledge of the student in difficult concepts of the taught modules. The following questions and possible answers were considered:

Question 1: Did you find that the virtual labs on Mobile Communications and Sensor Networks meet recent technological needs of the telecommunication and IT sec- 
tor? This question was used to quantify how the labs captured modern issues of telecommunications.

Question 2: Were the virtual labs useful for better understanding the related course modules? The question was used to capture the relevance of the exercises with the taught content.

Question 3: Would you recommend it to another student of another institution? It was used to observe the potential penetration of the labs to other institutions.

Question 4: Did you find the virtual labs easy to use? Used for quantifying the ease of use of the software package.

Question 5: Did you find the GUIs of the virtual labs easy to use and user friendly? It was used to observe if the GUIs are user friendly and facilitate their use.

Question 6: Did the GUI's of the different exercises incorporated repeating, reusable elements? It was used to observe if different laboratory environments incorporated similar components and thus did not require completely different practice to understand.

Question 7: Overall the virtual labs increased my interest in Mobile Communications and sensor networks. It was used to observe if the software package stimulated the interest for the specific module.

The possible answers to the above mentioned questions were 1-strongly disagree, 2-disagree, 3-neutral, 4-agree, 5strongly agree. The results are shown in Fig. 4 . It can be observed that despite the fact that most of the students were graduates of different departments, such as computer science, electrical engineering, mathematics, physics, technology management and electronics, the labs increased their interest in the modules and enhanced their knowledge of the taught subjects. An average score of 4.6 was observed.

It is also noted that the 12 volunteers had higher final grades in the Mobile Communication Networks module $(+1$ unit by average in an integer scale $1-10)$ than the ones that did not participate in the assessment process. This outcome may exemplary mean that:

- The laboratory spurred the interest of the $50 \%$ of the students, OR

- It was beneficial to the most committed students, which happened to be the $50 \%$ of their total number.

In general, the aforementioned lightweight assessment yielded a sense of positive contribution to the students' experience. The laboratory was thus adopted for permanent use in the course curriculum.

\section{A. Technical Deployment Experiences}

Adopting the MATLAB platform for the implementation of the presented software package resulted into important gains and losses. This section presents the encountered problems and proposed workarounds.

While MATLAB code makes for open, shorter and more understandable source code, licensing issues must be considered in advance. Academic licenses are provided by the manufacturing firm. However, seat limitations and potential budget shortages may require special handling. In all, the authors feel that the provided libraries and support makes up for the monetary cost. Nonetheless, migration to free MATLAB clones, such as OCTAVE, could also be considered. However, MATLAB is natively supported in every version of the Windows, Linux, Unix and

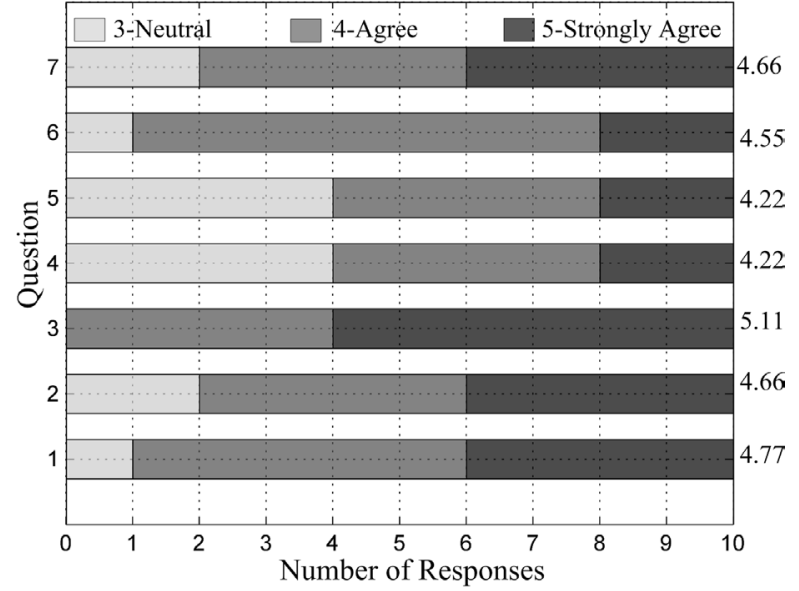

Figure 4. Evaluation of the vlabs from MSc students. The averages for each questions are presented on the right part of the figure.

Mac platforms. On the other hand, OCTAVE is Linux oriented (selected distributions only), and uses a layer of virtualization to work on Windows platforms. Thus, adopting OCTAVE may mean less market exposure and certainly slower execution times. It is clarified that the presented software package works on any platform that is supported by MATLAB. No special hardware is required.

A problem with the official MATLAB releases is the introduction of subtle changes into the programming interface in every new revision. While minor and generally easy to handle, these changes require continued maintenance of the software package. It is therefore proposed to strictly define the MATLAB revision required for operating the virtual laboratory. The $2009 \mathrm{~b}$ version was used for the complete presented package. Object oriented programming $(\mathrm{OO})$ is well supported in the MATLAB environment but deteriorates performance. In fact, an original heavily $\mathrm{OO}$ overall design underwent a major revision to resolve performance issues. Procedural programming was adopted in most aspects, while OO was limited to cases that required interfacing, such as adding new antennas and measurement types. The use of well supported parallel programming facilities alleviated the computational load in every notable case. Freely available GPGPU packages (general purpose graphics processing unit) may also be considered for further use.

Finally, the incorporation of cached data is an issue when their volume increases. Using the native .MAT format worked well for files up to $10 \mathrm{M}$ B in size. Arrays with roughly 100, 000 entries fall to this case. Greater sizes may turn problematic and should therefore be cached in memory in advance, either full or partially. Should there be no portability concerns, a dedicated SQL database can provide progressive, timely loading of data, improving the user experience.

\section{CONCLUSION}

A holistic virtual laboratory for wireless communications and sensor networks was presented. The software package aimed at combining the critical mass of explorative studies performed in the field over that past years. A structured educational and technical architecture provided complete and extensible coverage of classic and modern issues in the studied field. Real world assessment in the context of a postgraduate university course demonstrated the educational benefits of the proposed approach. 


\section{REFERENCES}

[1] T. Wolf, “Assessing Student Learning in a Virtual Laboratory Environment," IEEE Transactions on Education, vol. 53, no. 2, pp. 216-222, 2010.

[2] M. Duarte, B. P. Butz, S. M. Miller, and A. Mahalingam, “An Intelligent Universal Virtual Laboratory (UVL)," IEEE Transactions on Education, vol. 51, no. 1, pp. 2-9, 2008.

[3] C. Willems and C. Meinel, "Online assessment for hands on cyber security training in a virtual lab," in Proceedings of the 2012 IEEE Global Engineering Education Conference (EDUCON). IEEE, 2012, pp. 1-10.

[4] R. Adams, "The virtual oscilloscope: an impedance match to beginning ECE students," IEEE Transactions on Education, vol. 34, no. 4, pp. 350-356, 1991.

[5] M. Anisetti, V. Bellandi, A. Colombo, M. Cremonini, E. Damiani, F. Frati, J. T. Hounsou, and D. Rebeccani, "Learning Computer Networking on Open Paravirtual Laboratories," IEEE Transactions on Education, vol. 50, no. 4, pp. 302-311, 2007.

[6] V. M. Monthe, B. Batchakui, C. Tangha, and F. Tietche, "MVSYDIME: A virtual patient for medical diagnosis apprenticeship," in Proceedings of the 2012 IEEE Global Engineering Education Conference (EDUCON). IEEE, 2012, pp. 1-10.

[7] L. Edwards, "JHU Wireless Technology VLab," John Hopkins University, [Online] http://www.mathworks.com/products/matlab/ userstories.html?file $=10695,2011$.

[8] University of Illinois, "CNX Digital Communications VLab," [Online] http://courses.engr.illinois.edu/ece463/SP11/cnx.htm 2009.

[9] C. Dimakis, "Virtual Laboratory in Wireless Communications," [Online] http://vlabthlep.ee.auth.gr, 2009.

[10] University of St. Andrews, "WiFi Virtual Laboratory," [Online]: http://grid.cs.standrews.ac.uk/wine vl/wifi vl.html, 2010.

[11] T. Sturgeon, C. Allison, and A. Miller, "802.11 wireless experiments in a virtual world," ACM SIGCSE Bulletin, vol. 41, no. 3, p. 85, 2009.

[12] EDX Wireless, "EDX Signal Pro+Network Design," [Online] http://www.edx.com, 2012.

[13] Elanix Inc. "ELANIX System View", [Online] http://www.home.agilent.com, 2005.

[14] OPNET Technologies Inc., "OPNET Modeler Wireless Suite," [Online] http://www.opnet.com/solutions/networkrd/modeler.html, vol. 2012.

[15] LabVoltSystems Inc., "Analog/Digital Communications Training System," [Online] http://www.labvolt.com/products/telecommuni cations, vol. 2012.

[16] B. Barros, T. Read, and M. F. Verdejo, "Virtual Collaborative Experimentation: An Approach Combining Remote and Local Labs," IEEE Transactions on Education, vol. 51, no. 2, pp. 242 250, 2008.

[17] International Hellenic University, "IHU Virtual Laboratories," [Online] http://vlabs.ihu.edu.gr, 2010.

[18] MathWorks Inc, "MATLAB version (R2010a)," 2010. [Online]. Available: http://www.mathworks.com/products/matlab/

[19] John W. Eaton et al., "GNU Octave," 2012. [Online]. Available: http://www.gnu.org/software/octave/

[20] C. K. Liaskos, C. G. Koutitas, and I. Vlahavas, "Virtual Laboratorieson Wireless Communications: A Contemporary, Extensible
Approach," in Proceedings of the 3rd IEEE EDUCON conference on Engineering Education, Marrakesh, Morroco, April, 2012, pp. $1-10$.

[21] International Hellenic University, "MSc in Information and Communication Technology (ICT) Systems," 2012. [Online]. Available:

http://www.tech.ihu.edu.gr/index.php/courses/mastersmscs/mscini nformationandcommunicationtechnologyictsystems.html

[22] A. F. Molisch, Wireless communications, 2nd ed. Chichester and West Sussex and U.K: Wiley and IEEE, 2011.

[23] S. R. Saunders, Antennas and propagation for wireless communication systems. Chichester: Wiley \& Sons, 1999.

[24] G. Koutitas, "Green Network Planning of Single Frequency Networks," IEEE Transactions on Broadcasting, vol. 56, no. 4, pp. 541-550, 2010.

[25] G. Simon, P. Volgyesi, M. Maroti, and A. Ledeczi, "Simulationbased optimization of communication protocols for large scale wireless sensor networks", in 2003 IEEE Aerospace Conference Proceedings (Cat. No.03TH8652). IEEE, 2003, pp. 3_13393_1346.

[26] O. Dalle, G. Wainer, G. Stea, L. F. Perrone, P. M. Wightman, and M. A. Labrador, "Atarraya: a simulation tool to teach and research topology control algorithms for wireless sensor networks", in Proceedings of the Second International ICST Conference on Simulation Tools and Techniques. ICST, 2009.

[27] Web 3D Consortium, "The Virtual Reality Modeling Language (VRML) Standard," [Online] http://www.web3d.org/x3d/specifi cations/vrml/ISOIEC14772VRML97/, 2004.

[28] J. W. Eaton et al, "GNU Octave," [Online] http://www.gnu.org/software/octave, 2012.

[29] Accelereyes, "JACKETGPU Computing with MATLAB," [Online] http://www.accelereyes.com, 2012.

[30] MathWorks Inc,"Why is saving a large matrix to a MAT file very slow in MATLAB7.7?" 2011. [Online]. Available: http://www.mathworks.com/support/solutions/en/data/1APTWLQ/ index.html?product=SL\&solution=1APTWLQ

\section{AUTHORS}

Christos Liaskos is with the Department of Informatics, Aristotle University of Thessaloniki, Greece, email: cliaskos@csd.auth.gr.

George Koutitas is with the School of Science and Technology, International Hellenic University, Greece, email: g.koutitas@ihu.edu.gr

Ioannis Vlahavas is a professor at the Department of Informatics, Aristotle University and the Dean of School of Science and Technology, International Hellenic University, Greece, email: vlahavas@csd.auth.gr.

This research has been co-financed by the European Union (European Social Fund ESF) and Greek national funds through the Operational Program "Education and Lifelong Learning" MIS 200056 of the National Strategic Reference Framework (NSRF). The development of the application was proposed, managed and supervised by the legal authorities of the International Hellenic University, Thessaloniki, Greece. Submitted 16 January 2013. Published re-submitted by the authors 26 June 2013. 